\title{
Disorder-induced topology in quench dynamics
}

\author{
Hsiu-Chuan Hsu $\odot,{ }^{1,2,{ }^{*}}$ Pok-Man Chiu $\odot{ }^{3}$ and Po-Yao Chang $\odot^{3, \uparrow}$ \\ ${ }^{1}$ Graduate Institute of Applied Physics, National Chengchi University, Taipei 11605, Taiwan \\ ${ }^{2}$ Department of Computer Science, National Chengchi University, Taipei 11605, Taiwan \\ ${ }^{3}$ Department of Physics, National Tsing Hua University, Hsinchu 30013, Taiwan
}

(Received 27 January 2021; revised 23 August 2021; accepted 26 August 2021; published 13 September 2021)

\begin{abstract}
We study the effect of strong disorder on topology and entanglement in quench dynamics. Although disorderinduced topological phases have been well studied in equilibrium, the disorder-induced topology in quench dynamics has not been explored. In this paper, we predict a disorder-induced topology of postquench states characterized by the quantized dynamical Chern number and the crossings in the entanglement spectrum in $(1+$ 1) dimensions. The dynamical Chern number undergoes transitions from zero to unity and back to zero when increasing the disorder strength. The boundaries between different dynamical Chern numbers are determined by delocalized critical points in the postquench Hamiltonian with the strong disorder. An experimental realization in quantum walks is discussed.
\end{abstract}

DOI: 10.1103/PhysRevResearch.3.033242

\section{INTRODUCTION}

Topological phases of matter out of equilibrium and their phase transitions have attracted much theoretical and experimental interest. Their topological and nonequilibrium features have been demonstrated in various systems including ultracold atomic gases [1-4], quantum optics [5-7], superconducting qubits [8,9], and condensed matter systems [10-18]. Among these, topological Floquet systems have been widely studied [19-21]. These systems exhibit protected boundary states which are robust in the presence of disorder. More recently, topological phases in dynamical quench systems have been proposed [22-26]. For example, for a trivial state under a sudden quench by the Su-Schrieffer-Heeger ( $\mathrm{SSH}$ ) model, the topology of the postquench state is characterized by dynamical Chern numbers [22,24], the quantization of which describes a skyrmion texture of the postquench pseudospin in momentum-time space [27]. The topology in quench dynamics has been shown experimentally in photonic quantum walks [27-29] and superconducting qubits [30,31]. Moreover, the entanglement spectrum provides an additional probe of the topology. The robustness of crossings in the entanglement spectrum of the postquench states indicates the nontrivial topology in quench dynamics [23,24].

Besides the topological structures that emerge in quench dynamics, nontrivial topology can arise from disordered systems in equilibrium. In the strong disorder regime, an

\footnotetext{
*hcjhsu@nccu.edu.tw

†pychang@phys.nthu.edu.tw
}

Published by the American Physical Society under the terms of the Creative Commons Attribution 4.0 International license. Further distribution of this work must maintain attribution to the author(s) and the published article's title, journal citation, and DOI. unexpected topological phase with extensive boundary states is stabilized by the strong disorder. This phase is termed the topological Anderson insulator [32-36] and the transition between trivial and nontrivial phases is described by the delocalization criticality [37]. A generalization of the topological Anderson insulator to Floquet systems is proposed [38-41]. The strong disorder drives trivial Floquet systems into topological phases that host chiral edge modes coexisting with the localized bulk states in two-dimensional lattices. The transition also links to delocalization [42]. In constrast, quench Anderson disorder was studied theoretically in simple lattice models $[43,44]$. It has been shown that in the strong disorder regime, where the Anderson localization sets in, there is no sharp transition in the quench dynamics [43].

Although there are extensive studies in disorder-induced topology in Floquet systems, the effect of disorder on topology in quench dynamics is less discussed. It is shown that the crossings in the entanglement spectrum are robust against weak disorder and interactions [23]. However, it has not been known whether disorder could induce topology in quench dynamics.

In this paper, we demonstrate the strong disorder-induced topology in quench dynamics. We consider a quench protocol described by a trivial initial state (a fully pseudospin-polarized state) under a sudden quench by the SSH Hamiltonian in the presence of strong disorder. The topology of the postquench state is characterized by the dynamical Chern number which is zero/unity when the SSH model is trivial/nontrivial. We start at the clean limit where the postquench state is trivial. When the disorder strength is above the critical value, the postquench state has a quantized dynamical Chern number. The entanglement spectrum of the postquench states shows robust crossings which indicate the disorder-induced topology in quench dynamics. The postquench SSH Hamiltonian in this strong disorder regime has a disorder-induced winding number. The phase boundaries coincide with the transitions 

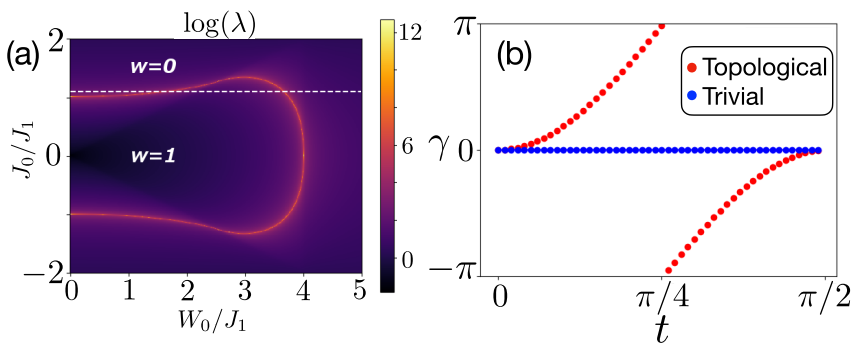

FIG. 1. (a) The phase diagram of the postquench Hamiltonian $H=H_{0}+H_{U}$. The white dashed line denotes $J_{0}=1.1$. (b) The time-dependent Berry phase in the clean limit. The blue dots are for the trivial postquench state $\left(J_{0} / J_{1}=1.1\right)$. The red dots are for the topological postquench state with a Berry phase flow from $t=0$ to $\pi / 2\left(J_{0} / J_{1}=0.5\right)$.

between vanishing and quantized dynamical Chern numbers. Our results demonstrate that the disorder-induced topology in quench dynamics in $(1+1)$ dimensions is directly related to the topological Anderson insulator.

\section{THE POSTQUENCH HAMILTONIAN}

We consider an eigenstate $\left|\Psi_{0}\right\rangle$ of a prequench Hamiltonian $H_{\text {pre }}$ at $t=0$ under a sudden quench by a postquench Hamiltonian $H_{\text {post }}$, and the postquench state is $|\Psi(t)\rangle=$ $\exp \left[-i H_{\text {post }} t\right]\left|\Psi_{0}\right\rangle$. We consider $H_{\text {post }}=H_{0}+H_{U}$, with

$$
\begin{aligned}
& H_{0}=\sum_{x=1}^{N_{x}} J_{0} c_{x, a}^{\dagger} c_{x, b}+J_{1} c_{x+1, a}^{\dagger} c_{x, b}+\text { H.c., } \\
& H_{U}=\sum_{x=1}^{N_{x}} U_{1 x} c_{x, a}^{\dagger} c_{x, b}+U_{2 x} c_{x, a}^{\dagger} c_{x+1, b}+\text { H.c. },
\end{aligned}
$$

where $H_{0}$ is the SSH Hamiltonian and $H_{U}$ is the time-reversal and particle-hole symmetry preserving disorder. Here $x$ is the label of the unit cell, $N_{x}$ is the total number of the unit cell. $c_{x a(b)}^{\dagger}, c_{x a(b)}$ are the creation and annihilation operators on sublattices $a, b$ on the $x$ th unit cell. $J_{0(1)}$ denotes the intracell (intercell) coupling, and $U_{1(2) x}$ is the random intracell (intercell) coupling strength given by the random number in the uniform distribution $\left[-W_{1(2)} / 2, W_{1(2)} / 2\right]$. We choose the disorder strengths $W_{1}=2 W_{2}=W_{0}$. The postquench Hamiltonian $H_{\text {post }}$ has the time-reversal symmetry $T: c_{x a(b)} \rightarrow c_{x a(b)}$, $i \rightarrow-i$, and the particle-hole symmetry $C: c_{x a(b)} \rightarrow c_{x b(a)}$, $i \rightarrow-i$, i.e., it belongs to the BDI symmetry class, $T^{2}=$ $C^{2}=1$

The topology of the postquench Hamiltonian $H_{\text {post }}$ in the presence of strong disorder is characterized by the winding number $W$ and the phase diagram is shown in Fig. 1(a). In the clean limit with $\left(J_{0} / J_{1}, W_{0} / J_{1}\right)=(1.1,0)$, the winding number is zero. When the disorder strength increases, the winding number becomes unity when $W_{0} / J_{1} \gtrsim 1.7$ and is back to zero when $W_{0} / J_{1} \gtrsim 3.6$ [the white dash in Fig. 1(a)]. This behavior demonstrates the disorder-induced quantized winding number in the postquench Hamiltonian and is referred to as a topological Anderson insulator [45]. The phase boundaries of the trivial and the topological Anderson insulating phases are obtained by the divergence of the localization length $\lambda$ [37] [see Appendix A]:

$$
\frac{1}{\lambda}=\left|\ln \left[\frac{\left|2 J_{1}+W_{1}\right|^{\frac{J_{1}}{W_{1}}+\frac{1}{2}}\left|2 J_{0}-W_{2}\right|^{\frac{J_{0}}{W_{2}}-\frac{1}{2}}}{\left|2 J_{1}-W_{1}\right|^{\frac{J_{1}}{W_{1}}-\frac{1}{2}}\left|2 J_{0}+W_{2}\right|^{\frac{J_{0}}{W_{2}}+\frac{1}{2}}}\right]\right| .
$$

\section{THE QUENCH PROTOCOL}

In the clean limit, the postquench Hamiltonian is diagonalized in the momentum space $H_{\text {post }}=\sum_{k} \psi_{k}^{\dagger} \mathcal{H}_{\text {post }}(k) \psi_{k}$ with $\psi_{k}=\left(c_{k a}, c_{k b}\right)^{\mathrm{T}}$ with eigenenergies $\pm|E(k)|$. Since each single-particle state does not interact with each other, the single-particle state evolves individually $|\psi(k, t)\rangle=$ $e^{-i \mathcal{H}_{\text {post }}(k) t}\left|\psi_{0}(k)\right\rangle$, where $\left|\psi_{0}(k)\right\rangle$ is the single-particle ground state of the prequenched single-particle Hamiltonian $\mathcal{H}_{\text {pre }}(k)$. For each individual postquench single-particle state, the period of the dynamics is $T_{k}=2 \pi /|E(k)|$. The set of singleparticle states $|\psi(k, t)\rangle$ have a corresponding momentum-time manifold $k \in[0,2 \pi], t_{k} \in\left[0, T_{k}\right]$ which is a momentumtime torus. This torus is distorted because different $k$ has different circumference $T_{k}$. Since the deformation of the distorted torus to a ordinary torus (same circumference) does not change the topology, one can rescale the period of the dynamics to be $T_{k}=2 \pi$ The rescaling of the period is equivalent to flattening the postquench Hamiltonian, $\mathcal{H}^{F}(k)=$ $\mathcal{H}_{\text {post }}(k) /\left|E_{(k)}\right|$. We focus on the flattened Hamiltonian which allows us to construct the effective Hamiltonian $\mathcal{H}_{\text {eff }}(k, t)=$ $e^{-i \mathcal{H}^{F}(k) t} \mathcal{H}_{\text {pre }}(k) e^{i \mathcal{H}^{F}(k) t}$ for analyzing the topological property of the postquench dynamics [see Appendix B].

\section{A. Different prequench Hamiltonians}

The postquench state has two inputs, the prequench Hamiltonian $\mathcal{H}_{\text {pre }}(k)$ and the postquench Hamiltonian $\mathcal{H}_{0}(k)$. If the prequench and the postquench Hamiltonians are in the same symmetry class (BDI), the topology of the postquench state is characterized by the dynamical Chern number in the half of the Brillouin zone (BZ), $k \in[0, \pi]$ and $t \in[0, \pi][22]$. However, the dynamical Chern number is vanishing in the full $\mathrm{BZ}, k \in[0,2 \pi]$ and $t \in[0, \pi]$. To study the disorder-induced topology in quench dynamics, the real-space formalism is needed and requires the information of the full BZ. Since the dynamical Chern number vanishes in the full BZ and $t \in[0, \pi]$, no disorder-induced topology can happen in this quench protocol. On the other hand, if the prequench Hamiltonian $\mathcal{H}_{\text {pre }}(k)=-\sigma_{z}$ which is not in the same symmetry class as the postquench Hamiltonian, the dynamical Chern number is quantized in the full BZ, $t \in[0, \pi / 2]$ [24] [see Appendix B]. This prequench Hamiltonian allows us to formulate the dynamical Chern number in real space and study the disorder-induced topology.

In this case, the single-particle state is fully pseudospin polarized and the real-space expression is $\left|\psi_{i}\right\rangle=(1,0)^{\mathrm{T}} \otimes|i\rangle$, where $(1,0)^{\mathrm{T}}$ denotes one particle at the sublattice $a,|i\rangle=$ $(0, \ldots, 1, \ldots, 0)^{\mathrm{T}}$ denotes the only nonvanishing $i$ th element with $i$ being the site label $i=1 \ldots N_{x}$. The postquench Hamiltonian in the presence of disorder can be flattened by using the projectors, $\mathcal{H}^{F}=\left|\psi_{+}\right\rangle\left\langle\psi_{+}|-| \psi_{-}\right\rangle\left\langle\psi_{-}\right|$, where $\left|\psi_{ \pm}\right\rangle$are the eigenstates of $\mathcal{H}$ with positive/negative energies. 


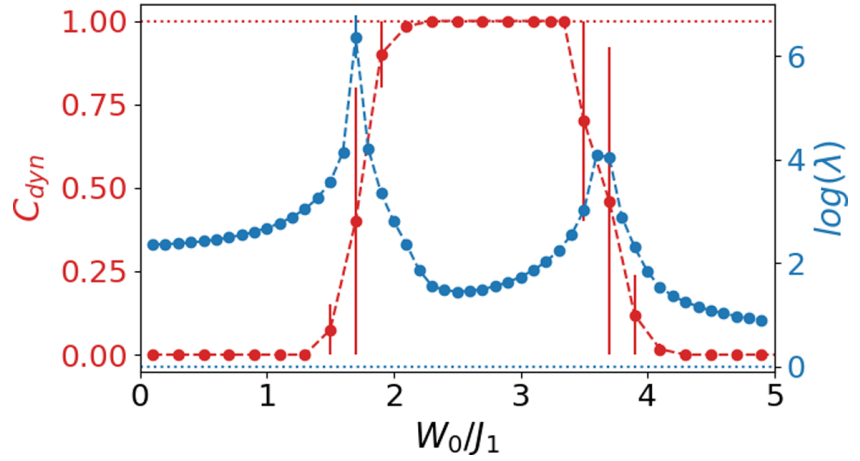

FIG. 2. The disorder-average dynamical Chern number and the localization length for the postquench Hamiltonian. The error bar is the standard deviation. There are more than 20 disorder realizations for each data point. The parameters are $J_{0} / J_{1}=1.1, N_{x}=400$.

\section{B. Berry phase and dynamical Chern number}

To determine the dynamical Chern number in the real space, we compute the Berry phase with the twisted boundary condition $[46,47]$ by the overlap matrix [48-50]. The overlap matrix at a $t$ is defined as $M_{i j}^{\ell}(t)=\left\langle\psi_{i}^{\theta_{\ell}}(t) \mid \psi_{j}^{\theta_{\ell+1}}(t)\right\rangle$, where $\left|\psi_{i}^{\theta_{\ell}}(t)\right\rangle=\exp \left[-i \mathcal{H}_{\text {post }}^{\theta_{\ell}} t\right]\left|\psi_{i}\right\rangle, i$ is the index of the single-particle state, and $\mathcal{H}_{\text {post }}^{\theta_{\ell}}$ is the flattened postquench Hamiltonian with twisted boundary phase $\theta_{\ell}=\frac{2 \pi \ell}{L}$ [49], where $L$ is the number of mesh points and $l=1, \cdots, L$. The Berry phase is given by $\gamma(t)=\operatorname{Im}\left[\ln \operatorname{det} \prod_{\ell=1}^{L} M^{\ell}(t)\right]$. The Berry phase as a function of $t$ has no jump when the postquench state is trivial [Fig. 1(b) blue dots]. In contrast, when the postquench state is topological, the Berry phase flow has $2 \pi$ jumps at $t=\pi / 4$ as shown by the red dots in Fig. 1(b). The Wannier center flow also shows similar behavior which we demonstrate in the Appendix C.

The dynamical Chern number is obtained by integrating the time derivative of the Berry phase $C_{\text {dyn }}=\frac{1}{2 \pi} \int_{0}^{\pi / 2} d t \frac{\partial \gamma(t)}{\partial t}$. Since $t=\pi / 2$ is the time taken for the pseudospin to precess from the north pole to the south pole, the integration is equivalent to counting the numbers of the pseudospin $\hat{n}_{i}(t)=$ $\left\langle\psi_{i}(t)|\vec{\sigma}| \psi_{i}(t)\right\rangle$ wrapping around the entire Bloch sphere [24]. The disorder-induced dynamical Chern number is shown in the red dots in Fig. 2(a). In the weak disorder limit, $W_{0} / J_{1} \lesssim$ 2.2 and $J_{0} / J_{1}=1.1$, the dynamical Chern number vanishes. While increasing the disorder strength $W_{0}$, the dynamical Chern number is quantized with negligible fluctuations in the region $2.2 \lesssim W_{0} \lesssim 3.2$. This behavior demonstrates that the disorder drives the trivial postquench state to be topological, and we refer to it as the disorder-induced topology in quench dynamics.

The phase boundaries of the zero and unity dynamical Chern numbers coincide with the phase boundaries of the postquench Hamiltonian obtained from the divergence of the localization length [the white dashed line in Fig. 1(a) and the blue dots in Fig. 2]. It was demonstrated that in the clean limit, the topology of the quench dynamics is related to that of the postquench Hamiltonian $[23,24]$. Here, we observe that the relation is still held for the disorder-induced topology.

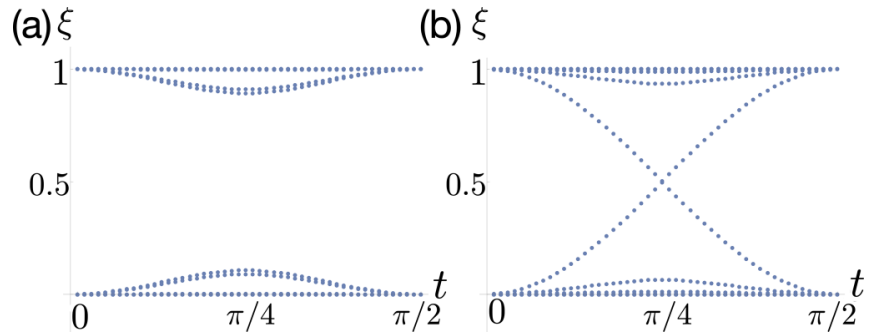

FIG. 3. The entanglement spectrum of the postquench state with the bipartition $l_{A}=l_{B}=N_{x} / 2$, where $l_{A(B)}$ is the length of the subsystem $A(B)$ and $N_{x}$ is the length of the total system. The parameters are $J_{0} / J_{1}=1.1$. (a) $W_{0}=0$ (clean limit). (b) $W_{0}=3$. There are 100 disorder realizations for each data point.

\section{Entanglement spectrum}

The entanglement spectrum provides additional information of the topology induced by disorder in quench dynamics. It is shown that the crossings in the entanglement spectrum reveal the topological properties in both the equilibrium systems [51-57] and out-of-equilibrium systems $[23,24,58,59]$. The presence/absence of the robust crossings in the entanglement spectrum indicates the postquench state is topological/trivial. To compute the entanglement properties, the system is bipartite spatially into $A$ and $B$ subsystems, where the postquench many-body state is expressed as $|\Psi(t)\rangle=\sum_{i, j} C_{i j}(t)\left|A_{i}\right\rangle\left|B_{j}\right\rangle$, with $\left|A(B)_{i}\right\rangle$ being the local basis in subsystem $A(B)$. We can compute the reduced density matrix $\rho_{A}(t)=\operatorname{Tr}_{B}|\Psi(t)\rangle\langle\Psi(t)|=\frac{1}{N} e^{-H_{A}(t)}$, where $H_{A}(t)$ is referred to as the entanglement Hamiltonian, $N$ is the normalization constant, and the spectrum of $H_{A}(t)$ is the entanglement spectrum.

In free-fermion systems, the eigenvalues of the reduced density matrix can be obtained from the correlation matrix $C_{\mathbf{x}, \mathbf{x}^{\prime}}(t)=\left\langle\Psi(t)\left|c_{\mathbf{x}}^{\dagger} c_{\mathbf{x}^{\prime}}\right| \Psi(t)\right\rangle=\sum_{i}\left|\psi_{i}\left(\mathbf{x}^{\prime}, t\right)\right\rangle\left\langle\psi_{i}(\mathbf{x}, t)\right|$, where $\left|\psi_{i}(\mathbf{x}, t)\right\rangle$ is the postquench single-particle state [see Appendix D]. The spectrum $\xi(t)$ of the correlation matrix $C_{\mathbf{x}, \mathbf{x}^{\prime}}(t)$ with $x, x^{\prime}$ being restricted in $A$ is related to the entanglement spectrum $\epsilon(t)$ by $\xi(t)=1 /\left(1+e^{\epsilon(t)}\right)$ [60]. For simplicity, we refer $\xi(t)$ to the entanglement spectrum.

In the clean limit at $J_{0} / J_{1}=1.1$ [Fig. 3(a)], the postquench state is trivial and there are no crossings in the entanglement spectrum $\xi(t)$. When the disorder strength is above the critical values, the entanglement spectrum $\xi(t)$ of the postquench state shows a crossing at $t=\pi / 4$ [Fig. 3(b)]. The existence of the crossings in the entanglement spectrum agrees with the nonvanishing dynamical Chern number of the postquench state. We demonstrate the nonvanishing dynamical Chern number and the crossings in the entanglement spectrum for other parameters in Appendix E.

\section{EXPERIMENTAL REALIZATION}

Discrete-time quantum walks are great platforms for simulating the topological phases of matter [28,61,62], quantum quenches [27,29], and disorder phenomena [63-65]. Following Ref. [27], the discrete-time evolution operator for a one-dimensional lattice with single photons can be engineered by the cascaded half-wave plates and beam displacers. 


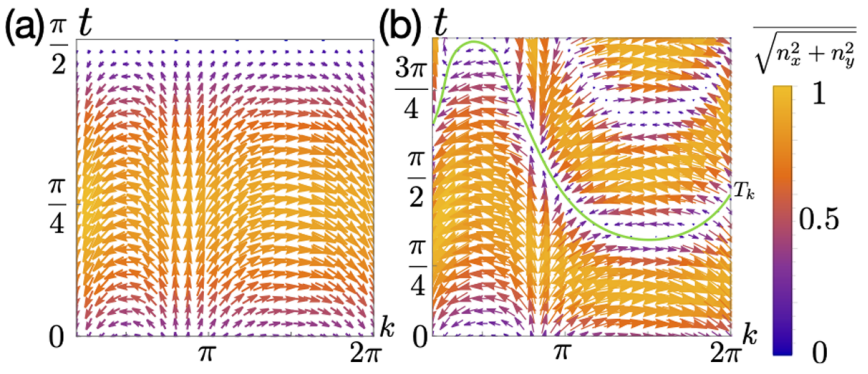

(c)

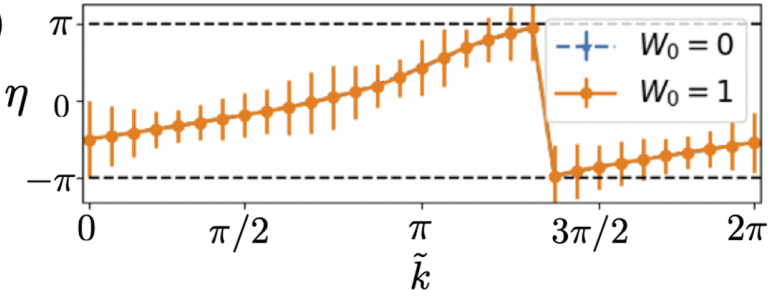

FIG. 4. The postquench psuedospin texture in the momentumtime space without disorder with $J_{0} / J_{1}=0.5$ for (a)flattened Hamiltonian, (b) nonflattened Hamiltonian. The postquench psuedospin forms the Skyrmion texture in the momentum-time domain, $k \in[0,2 \pi], t \in[0, \pi / 2]$ in (a) and $t \in\left[0, T_{k}\right]$ in (b) where $T_{k}=$ $\pi /(2 E(k))$ is shown by the green line. (c) The long-time average of $\eta$ for the nonflattened Hamiltonian with $J_{0} / J_{1}=0.5$. Two curves almost overlap. The error bars are the standard deviation for 400 disorder realizations. $N_{x}=31$.

The Hilbert space is spanned by the polarization states $\left\{\left|P_{+}\right\rangle,\left|P_{-}\right\rangle\right\}$and the position state $|x\rangle$ with $x \in \mathbb{Z}$. The corresponding evolution operator for each time step is $U=$ $R\left(\phi_{1} / 2\right) S R\left(\phi_{2}\right) S R\left(\phi_{1} / 2\right)$, where $R(\phi)$ rotate the polarization by $\phi$ with respect to $y$ axis, and $S$ is the shift operator $S=$ $\sum_{x}|x-1\rangle\left\langle x|\otimes| P_{+}\right\rangle\left\langle P_{+}|+| x+1\right\rangle\left\langle x|\otimes| P_{-}\right\rangle\left\langle P_{-}\right|$. The polarization angle $\phi_{1(2)}(x)$ is spatially dependent and disorder can be introduced by choosing different $\phi_{1(2)}(x)$ for different positions $x$.

In a translation-invariant case, the unitary operator can be diagonalized in the momentum space and the effective Hamiltonian describing the pre/postquench system has the form $\mathcal{H}_{\mathrm{eff}}(k)=-i \ln U(k)$. It is shown that this quantum walk protocol [27] can simulate a sudden quench between $\mathcal{H}_{\text {eff }}^{\mathrm{i}}(k)$ and $\mathcal{H}_{\mathrm{eff}}^{\mathrm{f}}(k)$ of the SSH model. Here $\mathcal{H}_{\mathrm{eff}}^{\mathrm{i}}(k)$ and $\mathcal{H}_{\mathrm{eff}}^{\mathrm{f}}(k)$ are referred to as prequench and postquench Hamiltonians. The topology of the postquench state can be extrapolated from the postquench pseudospin $\mathbf{n}(k, t)=\operatorname{Tr}[\rho(k, t) \boldsymbol{\sigma}]$ with $\rho(k, t)=\left|\psi_{k}(t)\right\rangle\left\langle\psi_{k}(t)\right|$. The postquench pseudospin forms the Skyrmion texture in the momentum-time domain when the postquench state has non-vanishing dynamical Chern number [Fig. 4(a)]. The Skyrmion texture can be understood as the pseudospin pointing along the $+(-) z$ direction at $t=0(\pi / 2)$ and rotating clockwise as a function of $k$ on the $x-y$ plane. In the experimental setup, the Hamiltonian is non-flatten and the period of dynamics of each momentum is $T_{k}=\pi /(2 E(k))$. Nevertheless, the Skyrmion texture of the pseudospin can be observed in the momentum-time domain $k \in[0,2 \pi], t_{k} \in$ $\left[0, T_{k}\right][$ Fig. 4(b)] and was measured experimentally in the quantum walk setup [27].

In the presence of disorder, the momentum is no longer a good quantum number and the momentum-dependent period

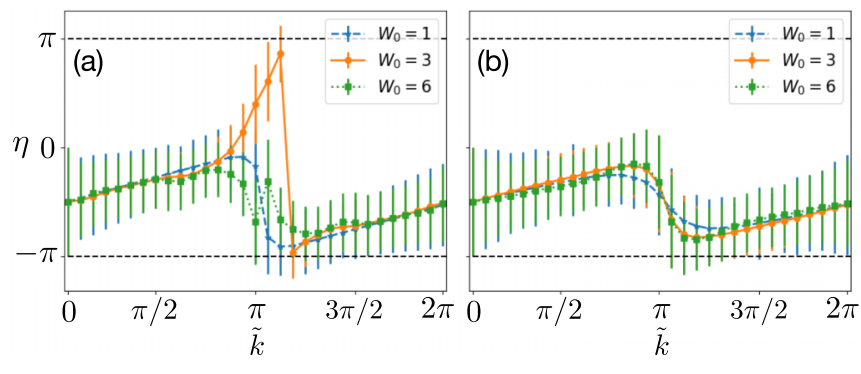

FIG. 5. The long-time average of $\eta$ for the postquench psuedospin in the pseudomomentum space given by nonflattened Hamiltonian with (a) $\frac{J_{0}}{J_{1}}=1.1$, and (b) $\frac{J_{0}}{J_{1}}=1.5$. The error bars are the standard deviations for 400 disorder realizations. $N_{x}=31$.

is not well-defined. For the nonflattened postquench Hamiltonian, we propose to measure the long-time average of the pseudospins $\overline{\left\langle\sigma_{i}\right\rangle_{T}}=\frac{1}{T} \int_{0}^{T} d t \overline{\left\langle\sigma_{i}\right\rangle}$, where $\left\langle\sigma_{i}\right\rangle=\operatorname{Tr}\left[\rho^{\prime}(\tilde{k}, t) \sigma_{i}\right]$ and

$$
\rho^{\prime}(\tilde{k}, t)=\overline{\frac{1}{2} \sum_{i=0}^{3} \sum_{x_{1}, x_{2}} e^{-i \tilde{k}\left(x_{1}-x_{2}\right)}\left\langle\psi_{x_{1}}(t)\left|\sigma_{i}\right| \psi_{x_{2}}(t)\right\rangle \sigma_{i} .}
$$

$\rho^{\prime}(\tilde{k}, t)$ is the disorder-averaged density matrix in the pseudomomentum-time space, where $\cdots$ denotes the disorder average. Here $\tilde{k}$ is referred to as the pseudomomentum, which indicates that the momentum is no longer a good quantum number in disordered systems. Since the $x, y$-components of the Skyrmion texture show a $2 \pi$ winding as a function of the pseudomomemtum $\tilde{k}$, one can monitor the in-plane pseudospin texture to detect the nontrivial topology by defining

$$
\eta=\operatorname{Im} \log \left[{\overline{\left\langle\sigma_{x}\right\rangle}}_{T}+i{\overline{\left\langle\sigma_{y}\right\rangle}}_{T}\right] .
$$

If the the postquench state is topological, $\eta$ shows a $2 \pi$ difference in $\tilde{k}=0$ to $2 \pi$.

We numerically show that $\eta$ can detect the topology of the postquench state in Figs. 4(c) and 5. The time taken for the average is $T=\pi / E_{\min }$, where $E_{\min }$ is the minimum absolute eigenenergy of the postquench Hamiltonian in the clean limit. This average time $T$ is the largest timescale in the system.

First, we demonstrate the topology of the postquench state is robust in the weak disorder region. As shown in Fig. 4(c), the in-plane pseudospin angle $\eta$ exhibits a $2 \pi$ winding in the clean limit $W_{0} / J_{1}=0$ and the weak disorder region $W_{0} / J_{1}=1$ for the parameter $J_{0} / J_{1}=0.5$. Next, we consider the disorder-induced topology for the parameter $J_{0} / J_{1}=1.1$. As we demonstrated previously, the postquench state is topological for the disorder strength $1.7 \lesssim W_{0} / J_{1} \lesssim 3.6$. As shown in Fig. 5(a), $\eta$ does not have a $2 \pi$ winding at $W_{0} / J_{1}=1$ and $W_{0} / J_{1}=6$, but exhibits a $2 \pi$ winding at $W_{0} / J_{1}=3$, reflecting the disorder-induced topology. In contrast, for the parameter $J_{0} / J_{1}=1.5$ which does not exhibit the disorder-induced topology, $\eta$ does not show a $2 \pi$ winding with different strong disorder strengths as shown in Fig. 5(b).

\section{CONCLUSION}

We predicted the disorder-induced topology in quench dynamics in $(1+1)$ dimensions. The topology is characterized by the dynamical Chern number and crossings in the 
entanglement spectrum. We showed the boundaries between trivial and nontrivial postquench states are identified by delocalized critical points in the postquench Hamiltonian. The quantized dynamical Chern number in $(1+1)$ dimensions corresponds to the winding number of the one-dimensional topological Anderson insulating phase of the SSH model. Finally, we proposed this phenomenon can be realized in quantum walk experiments.

\section{ACKNOWLEDGMENTS}

The authors thank Ching-Hao Chang and Chao-Cheng Kaun for hosting the workshop of quantum materials at Research Center for Applied Sciences, Academia Sinica, where the work was partially initiated. H.C.H. was supported by the Ministry of Science and Technology (MOST) in Taiwan, MOST 108-2112-M-004-002-MY2. P.-Y.C. was supported by the Young Scholar Fellowship Program under MOST. This work was supported by the MOST under Grant No. 110-2636-M-007-007.

\section{APPENDIX A: LOCALIZATION LENGTH}

When electrons are localized, the wave function exponentially decays with length, i.e., $\phi_{L} \propto e^{-L / \lambda}$, where $\phi_{L}=$ $\sum_{n=1}^{L}\left(\phi_{n a}, \phi_{n b}\right)^{T} c_{n}^{\dagger}$ is the eigenstate of the Hamiltonian $H=$ $H_{o}+H_{U}$ with length $n, \phi_{n a / b}$ are the coefficients for sublattice $a / b$ at site $n$ and $\lambda$ is the localization length. The Schrodinger equation for zero eigenenergy state becomes

$$
\begin{aligned}
& \left(J_{0}+U_{1 n}\right) \phi_{n b}+\left(J_{1}+U_{2 n}\right) \phi_{n-1, b}=0, \\
& \left(J_{0}+U_{1 n}\right) \phi_{n a}+\left(J_{1}+U_{2 n}\right) \phi_{n+1, a}=0 .
\end{aligned}
$$

The above equations give the ratio of coefficients between the first and the last site, $\left|\phi_{L a}\right|=\prod_{n=1}^{L}\left|\frac{J_{1}+U_{2 n}}{J_{0}+U_{1 n}} \phi_{1 a}\right|$ and $\left|\phi_{L b}\right|=$ $\prod_{n=1}^{L}\left|\frac{J_{0}+U_{1 n}}{J_{1}+U_{2 n}} \phi_{1 b}\right|$ for each sublattice, respectively. The final localization length for the system is the minimum of that of the sublattices. Thus, the localization length is given by

$$
\frac{1}{\lambda}=\frac{1}{L} \ln \prod_{n=1}^{L}\left|\frac{J_{1}+U_{2 n}}{J_{0}+U_{1 n}}\right| .
$$

The equation can be solved analytically [37].

Another approach to calculate the localization length is via Green's function. The localization length $\lambda$ is defined by

$$
\frac{2}{\lambda}=-\lim _{L \rightarrow \infty} \frac{1}{L} \operatorname{Tr} \ln \left|G_{1, L}\right|^{2},
$$

where $n$ is the total number of sites of the one-dimensional Hamiltonian, $G_{1, L}$ is the propagator connecting the first and last slice of the system [66]. $G_{1, n}$ is computed with the iterative Green's function method [66-68] by computing the on-site Green's function $G_{n, n}=\left(E-h_{n}-U_{f} G_{n-1, n-1} U_{b}\right)$ and $G_{1, n}=G_{1, n-1} U_{b} G_{n, n}$ recursively till $n$ is large enough for convergence, where $h_{n}=\left(J_{0}+U_{1, n}\right) \sigma_{x}, U_{f(b)}=\left(J_{1}+U_{2 n}\right)$ $\left(\sigma_{x}+(-) i \sigma_{y}\right) / 2$ and $U_{1(2) n}$ are defined in the main text.

Within this method, the Hamiltonian is constructed in a slicing scheme, i.e.,

$$
H_{N}=\sum_{i=1}^{N}\left(|i\rangle h_{i}\langle i|+| i\rangle U_{b}\langle i+1|+| i+1\rangle U_{f}\langle i|\right),
$$

for the system with $N$ slices, where $|i\rangle$ is the state for the $i$ th slice, $U_{f(b)}$ is the forward (backward) hopping matrices between the neighboring slices, and to calculate the Green's function for the system with $N+1$ slices, the Hamiltonian for $N+1$ slices is

$$
H_{N+1}=H_{N}+|N+1\rangle h_{N+1}\langle N+1|+H^{\prime},
$$

where $h_{N+1}$ is the Hamiltonian for $N+1$-th slices, the hopping matrix $H^{\prime}=|N\rangle U_{b}\langle N+1|+| N+1\rangle U_{f}\langle N|$ between the $N-$ th and $N+1-$ th slice is treated as a perturbing term to $H_{N}+|N+1\rangle h_{N+1}\langle N+1|$. According to the Dyson equation, the perturbed Green's function is given by $G_{N+1}=G_{o}+$ $G_{o} H^{\prime} G_{N+1}$, where $G_{o}=G_{N}+|N+1\rangle\left(E-h_{N+1}\right)^{-1}\langle N+1|$. Substituting $H^{\prime}$ into the Dyson equation, one obtains the Green's function for $N+1$ slices $\left(G_{N+1}\right)$ in which the submatrices are given by

$$
\begin{gathered}
\left\langle N+1\left|G_{N+1}\right| N+1\right\rangle=\left(E-h_{N+1}-U_{f}\left\langle N\left|G_{N}\right| N\right\rangle U_{b}\right)^{-1}, \\
\left\langle 1\left|G_{N+1}\right| N+1\right\rangle=\left\langle 1\left|G_{N}\right| N\right\rangle U_{b}\left\langle N+1\left|G_{N+1}\right| N+1\right\rangle .
\end{gathered}
$$

Equations (A7) and (A8) are the main iterative equations for obtaining the localization length shown in Appendix E.

\section{APPENDIX B: SYMMETRY ANALYSIS AND TOPOLOGICAL CLASSIFICATION}

The flattened Hamiltonian formalism allows us to construct the effective Hamiltonian,

$$
\mathcal{H}_{\text {eff }}(k, t)=e^{-i \mathcal{H}_{0}^{F} t} \mathcal{H}_{\text {pre }}(k) e^{i \mathcal{H}_{0}^{F} t} .
$$

The topological invariants can be classified according to the symmetries of the effective Hamiltonian. For the prequench Hamiltonian $\mathcal{H}_{\text {pre }}(k)=-\sigma_{z}$ and the postquench Hamiltonian
$\mathcal{H}_{0}(k)=h_{x}(k) \sigma_{x}+h_{y}(k) \sigma_{y}$, one has the effective Hamiltonian

$$
\begin{aligned}
\mathcal{H}_{\text {eff }}(k, t)= & \frac{h_{y}(k) \sin 2 t}{\sqrt{h_{x}(k)^{2}+h_{y}(k)^{2}}} \sigma_{x} \\
& -\frac{h_{x}(k) \sin 2 t}{\sqrt{h_{x}(k)^{2}+h_{y}(k)^{2}}} \sigma_{y}+\cos 2 t \sigma_{z} .
\end{aligned}
$$

The effective Hamiltonian breaks the particle-hole symmetry explicitly, but preserves the time-reversal symmetry $\mathcal{T} \mathcal{H}_{\text {eff }}(k, t) \mathcal{T}^{-1}=\mathcal{H}_{\text {eff }}(-k,-t)$, and the additional two twofold symmetries $\sigma_{z} \mathcal{H}_{\text {eff }}(k, t) \sigma_{z}=\mathcal{H}_{\text {eff }}(k,-t)$, $\sigma_{x} \mathcal{H}_{\text {eff }}(k, t) \sigma_{x}=-\mathcal{H}_{\text {eff }}(-k, t)$. These two additional 
symmetries together with the time-reversal symmetry lead to a $\mathbb{Z}$ classification in $(1+1)$ dimensions. The former twofold symmetry acts like the reflection symmetry in the time domain. There are two fixed points $t=0$ and $\pi / 2$ such that $\left[\sigma_{z}, \mathcal{H}_{\text {eff }}(k, 0)\right]=\left[\sigma_{z}, \mathcal{H}_{\text {eff }}(k, \pi / 2)\right]=0$. The dynamical Chern number in this effective Hamiltonian is quantized in the half of the momentum-time space $k \in[0,2 \pi]$, $t \in[0, \pi / 2]$ [69-72].

The effective Hamiltonian has the following symmetries:

$$
\begin{gathered}
\mathcal{T} \mathcal{H}_{\mathrm{eff}}(k, t) \mathcal{T}^{-1}=\mathcal{H}_{\mathrm{eff}}(-k,-t), \\
\mathcal{R}_{t} H_{\mathrm{eff}}(k, t) \mathcal{R}_{t}^{-1}=\mathcal{H}_{\mathrm{eff}}(k,-t), \\
\mathcal{M}_{x} \mathcal{H}_{\mathrm{eff}}(k, t) \mathcal{M}_{x}^{-1}=-\mathcal{H}_{\mathrm{eff}}(-k, t),
\end{gathered}
$$

where $\mathcal{T}^{2}=\mathcal{R}_{t}^{2}=\mathcal{M}_{x}^{2}=1,\left\{\mathcal{R}_{t}, \mathcal{M}_{x}\right\}=0$, and $\left[\mathcal{T}, \mathcal{R}_{t}\right]=$ $\left[\mathcal{T}, \mathcal{M}_{x}\right]=0$.

The effective Hamiltonian can be expressed in terms of the effective massive Dirac Hamiltonian $\mathcal{H}_{\text {eff }}(k, t)=k \gamma_{1}+$ $t \gamma_{2}+M_{0} \gamma_{0}$, with $\left\{\gamma_{i}, \gamma_{j}\right\}=0(i=0,1,2)$. We construct the minimal effective Dirac Hamiltonian in terms of the tensor product form of the Pauli matrices

$$
\begin{aligned}
& \gamma_{1}=\sigma_{x} \otimes \sigma_{x}, \quad \gamma_{2}=\sigma_{y} \otimes \mathbb{I}_{2 \times 2}, \quad \gamma_{0}=\sigma_{z} \otimes \mathbb{I}_{2 \times 2}, \\
& \mathcal{T}=\mathbb{I}_{2 \times 2} \otimes \sigma_{z} \mathcal{K}, \quad \mathcal{R}_{t}=\sigma_{z} \otimes \sigma_{z}, \quad \mathcal{M}_{x}=\sigma_{x} \otimes \mathbb{I}_{2 \times 2} .
\end{aligned}
$$

One can check the only allowed mass term which preserving all the symmetries is the $\gamma_{0}$. For the $\mathbb{Z}$ classification, we need to make copies of the original effective Hamiltonian. For simplicity, we just make one copy. The double Hamiltonian is $\mathcal{H}_{\text {eff }}(k, t)=k \gamma_{1} \otimes \mathbb{I}_{2 \times 2}+t \gamma_{2} \otimes \mathbb{I}_{2 \times 2}+M_{0} \gamma_{0} \otimes \mathbb{I}_{2 \times 2}$, for which there are no other symmetry-preserving mass terms. This indicates that different phases are not adiabatically connected in this system. On the other hand, we can flip one momentum of the copy and construct the double Hamiltonian, $\mathcal{H}_{\text {eff }}(k, t)=k \gamma_{1} \otimes \sigma_{z}+t \gamma_{2} \otimes \mathbb{I}_{2 \times 2}+M_{0} \gamma_{0} \otimes \mathbb{I}_{2 \times 2}$. There is another symmetry-allowed mass term (anticommute with $\left.\gamma_{0} \otimes \mathbb{I}_{2 \times 2} \otimes \mathbb{I}_{2 \times 2}\right), M_{1}=\sigma_{y} \otimes \sigma_{y} \otimes \sigma_{y}$. This indicates the systems are all in the same phase. We conclude from the above analysis that the system belongs to a $\mathbb{Z}$ classification. Similar classification schemes can be found in Refs. [69-72].

\section{APPENDIX C: WANNIER CENTER WITH DISORDERS}

In translational invariant systems, the Wannier orbits are constructed from the Bloch states $u_{n \mathbf{k}}(\mathbf{r}), w_{n}(\mathbf{r}-\mathbf{R})=$ $\frac{1}{\Omega} \int d \mathbf{k} e^{i \mathbf{k} \cdot(\mathbf{r}-\mathbf{R})} u_{n \mathbf{k}}(\mathbf{r})$, with $\Omega$ being the volume of the system, $\mathbf{R}$ is the position of the unit cell, and $\mathbf{r}$ is the local position of the Wannier orbits within the unit cell. In an insulator, these Wannier orbits are localized states and are the eigenstates of the projected position operator $X_{P}=P X P$, where $P$ is the projector to the occupied states which are well-defined in an insulator.

To construct the Wannier orbits without using Bloch states, we first write down the Hamiltonian in the real space $\mathcal{H}_{I J}$, where $I(J)$ includes the band indices and positions. The spectrum can exhibit a gap and the corresponding occupied states $\left|\psi_{\alpha I}\right\rangle$ are well-defined. Here $\alpha$ is the eigenenergy index. The corresponding projectors are $P_{I J}=\sum_{\alpha \in \text { occ. }}\left|\psi_{\alpha I}\right\rangle\left\langle\psi_{\alpha J}\right|$. The position operator can be defined by as a diagonal
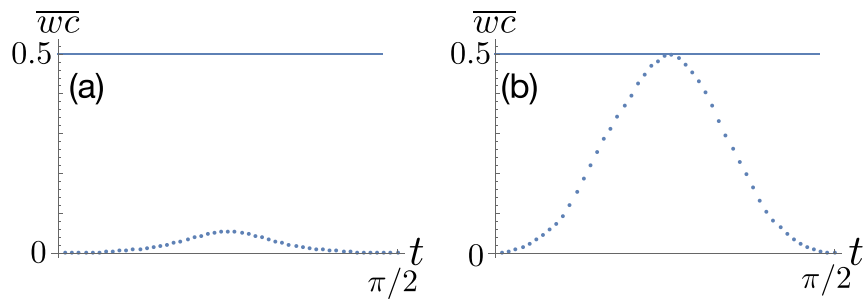

FIG. 6. The Wannier center as a function of $t$. (a) Disorderfree Hamiltonian $\left(\frac{J_{0}}{J_{1}}, \frac{W_{0}}{J_{1}}\right)=(1.1,0)$, (b) disordered Hamiltonian $\left(\frac{J_{0}}{J_{1}}, \frac{W_{0}}{J_{1}}\right)=(1.1,3)$. There are 100 disorder realizations.

matrix $\operatorname{diag}(1, \cdots, 1,2, \cdots 2, \cdots, N, \cdots, N)$, where $N$ is the total number of sites and at each site there are $L$ bands. The projected position operator can be constructed as usual $X_{P}=P X P[73]$.

Since the Wannier orbits are the eigenstates of the $X_{P}$, we can find diagonalize the $X_{P}$ and get the set of eigenstates. If the set of the eigenstates are localized states, then these states are the Wannier orbits and the corresponding eigenvalues are the position of the Wannier states. The Wannier center of a localized state in $M$-th site can be defined as $w c_{M}=$ $\left|\left\langle w_{M}\left|X_{P}\right| w_{M}\right\rangle-M\right|$. We have $0<\left\langle x_{M}\right\rangle<1$. We can further define the average Wannier center $\overline{w c}=\frac{1}{N} \sum_{m=1}^{N} w c_{M}$. In the presence of the chiral symmetry in one-dimensional gapped systems, the average Wannier center can have two values $\overline{w c}=0$ and 0.5 . The former corresponds to a trivial phase and the latter is the topological phase. Although in the quench setup, the effective Hamiltonian does not have the chiral symmetry, we observe the Wannier center of the topological postquench state reaches $\overline{w c}=0.5$ [Fig. 6(b)]. On the other hand, for the trivial postquench state, the Wannier center is below 0.5 [Fig. 6(a)].

\section{APPENDIX D: CORRELATION FUNCTION FORMALISM IN QUENCH SETUPS}

We consider an initial state contains $N$ particles. Each single-particle state we denote by $\left|\phi_{\alpha}(\mathbf{x})\right\rangle, \alpha=1, \ldots, N, \mathbf{x}$ is the internal degrees of freedom, including position, spin, and the band. We require these single-particle states are orthonormal, $\sum_{\mathbf{x}}\left\langle\phi_{\alpha}(\mathbf{x}) \mid \phi_{\beta}(\mathbf{x})\right\rangle=\delta_{\alpha, \beta}$. The $N$-particle initial state can be expressed as the Slater determinant of the single-particle state:

$$
\left|\Psi_{0}\right\rangle=\operatorname{Det}\left[\left|\phi_{i}\left(\mathbf{x}_{j}\right)\right\rangle\right], \quad i, j=1, \ldots, N .
$$

We consider an unitary evolution of this initial state $\left|\Psi_{0}\right\rangle$ by a static Hamiltonian $H=\sum_{\mathbf{x}, \mathbf{x}^{\prime}} \mathcal{H}_{\mathbf{x}, \mathbf{x}^{\prime}} c_{\mathbf{x}}^{\dagger} c_{\mathbf{x}^{\prime}}$, where $c_{\mathbf{x}}^{(\dagger)}$ is the annihilation (creation) operator. Each single-particle state under this evolution is $\left|\phi_{\alpha}(\mathbf{x}, t)\right\rangle=\sum_{\mathbf{x}^{\prime}} \exp \left[-i \mathcal{H}_{\mathbf{x}, \mathbf{x}^{\prime}} t\right]\left|\phi_{\alpha}\left(\mathbf{x}^{\prime}\right)\right\rangle, \alpha=$ $1, \ldots, N$. The postquench $N$-particle state is

$$
|\Psi(t)\rangle=e^{-i H t}\left|\Psi_{0}\right\rangle=\operatorname{Det}\left[\left|\phi_{i}\left(\mathbf{x}_{j}, t\right)\right\rangle\right]=\prod_{i} d_{i}^{\dagger}(t)|0\rangle,
$$

where

$$
\begin{aligned}
d_{i}^{\dagger}(t) & =e^{-i H t} d_{i}^{\dagger} e^{i H t}=e^{-i H t} \sum_{\mathbf{y}} V_{i \mathbf{y}} c_{\mathbf{y}}^{\dagger} e^{i H t} \\
& =\sum_{\mathbf{x}, \mathbf{y}} V_{i \mathbf{y}} U_{\mathbf{y}, \mathbf{x}}(t) c_{\mathbf{x}}^{\dagger},
\end{aligned}
$$




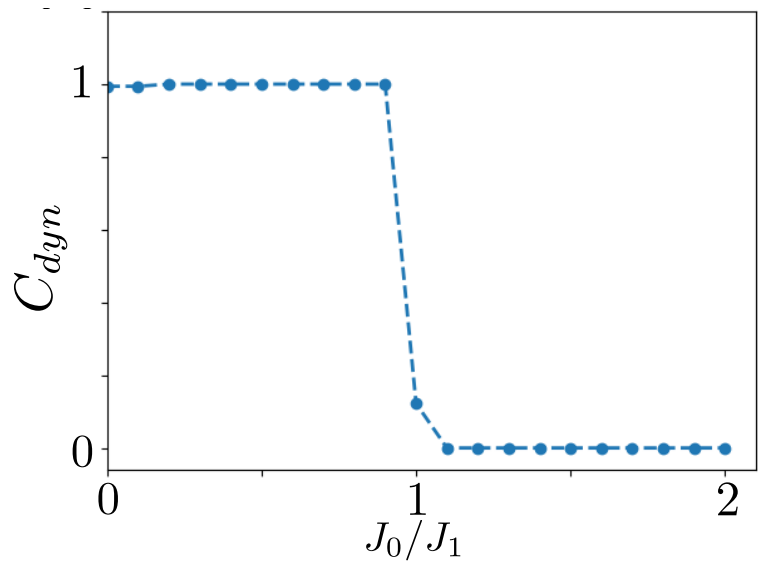

FIG. 7. The dynamical Chern number (DCN) for the static Hamiltonian in the clean limit. The parameters are $J_{0}=1.1$, $J_{1}=1, N_{x}=400, L=20$. Here $L$ is the number of mesh points for the twisted boundary condition.

with $U_{\mathbf{y}, \mathbf{x}}(t)=e^{-i \mathcal{H}_{\mathbf{y}, \mathbf{x}} t}$ and $V_{i \mathbf{y}}$ being an unitary matrix that rotates $d_{i}^{\dagger}$ to $c_{\mathbf{y}}^{\dagger}$.

The postquench single-particle state is

$$
d_{i}^{\dagger}(t)|0\rangle=\sum_{\mathbf{x}, \mathbf{y}} V_{i \mathbf{y}} U_{\mathbf{y}, \mathbf{x}}(t) c_{\mathbf{x}}^{\dagger}|0\rangle=\sum_{\mathbf{x}}\left|\phi_{i}(\mathbf{x}, t)\right\rangle .
$$

The correlation function constructed from the $N$-particle postquench state is

$$
\begin{aligned}
C_{\mathbf{x}, \mathbf{x}^{\prime}}(t)= & \left\langle\Psi(t)\left|c_{\mathbf{x}}^{\dagger} c_{\mathbf{x}^{\prime}}\right| \Psi(t)\right\rangle \\
= & \left.\left\langle 0\left|\prod_{\alpha} d_{\alpha} e^{i H t} c_{\mathbf{x}}^{\dagger} c_{\mathbf{x}^{\prime}} e^{-i H t} \prod_{\beta} d_{\beta}^{\dagger}\right| 0\right\rangle\right\rangle \\
= & \langle 0| \prod_{\alpha} d_{\alpha}\left[\sum_{\mathbf{y}, i} d_{i} U_{\mathbf{x}, \mathbf{y}}(t) V_{\mathbf{y} i}\right]^{\dagger} \\
& \left.\times\left[\sum_{\mathbf{y}^{\prime}, j} U_{\mathbf{x}^{\prime}, \mathbf{y}^{\prime}}(t) V_{\mathbf{y}^{\prime} j} d_{i}\right] \prod_{\beta} d_{\beta}^{\dagger}|| 0\right\rangle \\
= & \sum_{i}\left[\sum_{\mathbf{y}} U_{\mathbf{x}, \mathbf{y}}(t) V_{\mathbf{y} i}\right]^{\dagger}\left[\sum_{\mathbf{y}^{\prime}} U_{\mathbf{x}^{\prime}, \mathbf{y}^{\prime}}(t) V_{\mathbf{y}^{\prime} i}\right] \\
= & \sum_{i}\left|\phi_{i}\left(\mathbf{x}^{\prime}, t\right)\right\rangle\left\langle\phi_{i}(\mathbf{x}, t)\right| .
\end{aligned}
$$
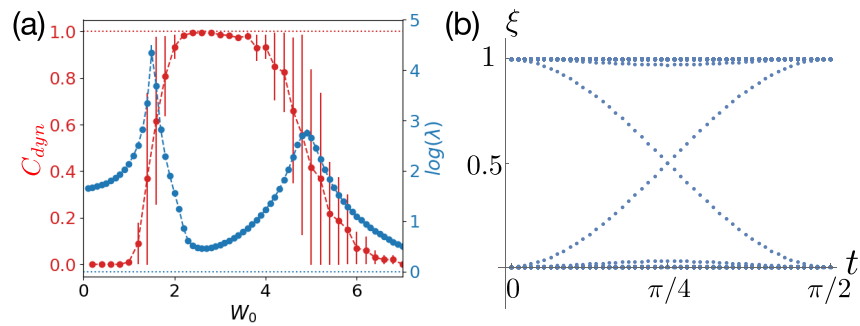

FIG. 8. (a) The disorder averaged mean dynamical Chern number and localization length obtained from Eq. (A4) for the quench Hamiltonian. The error bar is the standard deviation. The parameters are $J_{0}=1.1, J_{1}=1, N_{x}=100, L=400$. (b) The entanglement spectrum of the postquench state with $W_{1}=3$. The parameters are $J_{0}=1.1, J_{1}=1, N_{x}=400$. There are more than 50 disorder realizations for each data point.

The correlation matrix can be used for computing the entanglement spectrum. The existence of the crossings in the entanglement spectrum can detect the topology of the postquench state as demonstrated in several examples.

\section{APPENDIX E: OTHER PARAMETERS FOR THE DISORDER-INDUCED TOPOLOGY IN QUENCH DYNAMICS}

In the clean limit, the dynamical Chern numbers are calculated for $0 \leqslant J_{0} \leqslant 2$ and $J_{1}=1$ of the SSH Hamiltonian $H_{o}$. The results are shown in Fig. 7. For $J_{0}>1$, the static Hamiltonian becomes trivial and the dynamical Chern number is zero.

We consider the case with vanishing intercell disorder $W_{2}=0$. We find for $2.2 \lesssim W_{1} \lesssim 3.8$, the dynamical Chern number is close to an integer with vanishing fluctuations as shown in Fig. 8(a). The phase boundaries, where the dynamical Chern number is close to half-integer, are at $W_{0}=$ $1.5,4.9$. The localization length $\lambda$ also indicates delocalized transitions at the same values of $W_{0}$ [Fig. 8(a)]. The entanglement spectrum has a crossing at $t=\pi / 4$ when the postquench state has integer dynamical Chern number $W_{1}=3$ [Fig. 8(b)].
[1] M. S. Foster, V. Gurarie, M. Dzero, and E. A. Yuzbashyan, Quench-Induced Floquet Topological $p$-Wave Superfluids, Phys. Rev. Lett. 113, 076403 (2014).

[2] K. Plekhanov, G. Roux, and K. Le Hur, Floquet engineering of Haldane Chern insulators and chiral bosonic phase transitions, Phys. Rev. B 95, 045102 (2017).

[3] N. R. Cooper, J. Dalibard, and I. B. Spielman, Topological bands for ultracold atoms, Rev. Mod. Phys. 91, 015005 (2019).

[4] G. Salerno, H. M. Price, M. Lebrat, S. Häusler, T. Esslinger, L. Corman, J.-P. Brantut, and N. Goldman, Quantized Hall Conductance of a Single Atomic Wire: A Proposal Based on Synthetic Dimensions, Phys. Rev. X 9, 041001 (2019).
[5] M. C. Rechtsman, J. M. Zeuner, Y. Plotnik, Y. Lumer, D. Podolsky, F. Dreisow, S. Nolte, M. Segev, and A. Szameit, Photonic Floquet topological insulators, Nature (London) 496, 196 (2013).

[6] K. Wang, X. Qiu, L. Xiao, X. Zhan, Z. Bian, W. Yi, and P. Xue, Simulating Dynamic Quantum Phase Transitions in Photonic Quantum Walks, Phys. Rev. Lett. 122, 020501 (2019).

[7] T. Ozawa, H. M. Price, A. Amo, N. Goldman, M. Hafezi, L. Lu, M. C. Rechtsman, D. Schuster, J. Simon, O. Zilberberg, and I. Carusotto, Topological photonics, Rev. Mod. Phys. 91, 015006 (2019). 
[8] O. Kyriienko and A. S. Sørensen, Floquet Quantum Simulation with Superconducting Qubits, Phys. Rev. Appl. 9, 064029 (2018).

[9] D. Malz and A. Smith, Topological Two-Dimensional Floquet Lattice on a Single Superconducting Qubit, Phys. Rev. Lett. 126, 163602 (2021)

[10] T. Kitagawa, T. Oka, A. Brataas, L. Fu, and E. Demler, Transport properties of nonequilibrium systems under the application of light: Photoinduced quantum hall insulators without Landau levels, Phys. Rev. B 84, 235108 (2011).

[11] M. Ezawa, Photoinduced Topological Phase Transition and a Single Dirac-Cone State in Silicene, Phys. Rev. Lett. 110, 026603 (2013).

[12] A. Kundu, H. A. Fertig, and B. Seradjeh, Effective Theory of Floquet Topological Transitions, Phys. Rev. Lett. 113, 236803 (2014).

[13] B. Gulácsi and B. Dóra, From Floquet to Dicke: Quantum Spin Hall Insulator Interacting with Quantum Light, Phys. Rev. Lett. 115, 160402 (2015).

[14] A. Farrell and T. Pereg-Barnea, Photon-Inhibited Topological Transport in Quantum Well Heterostructures, Phys. Rev. Lett. 115, 106403 (2015)

[15] K. Takasan, A. Daido, N. Kawakami, and Y. Yanase, Laserinduced topological superconductivity in cuprate thin films, Phys. Rev. B 95, 134508 (2017).

[16] S. A. Owerre, Photoinduced topological phase transitions in topological magnon insulators, Sci. Rep. 8, 4431 (2018).

[17] A. Lubatsch and R. Frank, Evolution of floquet topological quantum states in driven semiconductors, Eur. Phys. J. B 92, 215 (2019).

[18] T. Oka and S. Kitamura, Floquet engineering of quantum materials, Annu. Rev. Condens. Matter Phys. 10, 387 (2019).

[19] T. Kitagawa, E. Berg, M. Rudner, and E. Demler, Topological characterization of periodically driven quantum systems, Phys. Rev. B 82, 235114 (2010).

[20] N. H. Lindner, G. Refael, and V. Galitski, Floquet topological insulator in semiconductor quantum wells, Nat. Phys. 7, 490 (2011).

[21] L. Jiang, T. Kitagawa, J. Alicea, A. R. Akhmerov, D. Pekker, G. Refael, J. I. Cirac, E. Demler, M. D. Lukin, and P. Zoller, Majorana Fermions in Equilibrium and in Driven Cold-Atom Quantum Wires, Phys. Rev. Lett. 106, 220402 (2011).

[22] C. Yang, L. Li, and S. Chen, Dynamical topological invariant after a quantum quench, Phys. Rev. B 97, 060304(R) (2018).

[23] Z. Gong and M. Ueda, Topological Entanglement-Spectrum Crossing in Quench Dynamics, Phys. Rev. Lett. 121, 250601 (2018).

[24] P.-Y. Chang, Topology and entanglement in quench dynamics, Phys. Rev. B 97, 224304 (2018).

[25] B. Zhu, Y. Ke, H. Zhong, and C. Lee, Dynamic winding number for exploring band topology, Phys. Rev. Research 2, 023043 (2020).

[26] H. Hu and E. Zhao, Topological Invariants for Quantum Quench Dynamics from Unitary Evolution, Phys. Rev. Lett. 124, 160402 (2020).

[27] K. Wang, X. Qiu, L. Xiao, X. Zhan, Z. Bian, B. C. Sanders, W. Yi, and P. Xue, Observation of emergent momentum-time skyrmions in parity-time-symmetric non-unitary quench dynamics, Nat. Commun. 10, 2293 (2019).
[28] F. Cardano, A. D’Errico, A. Dauphin, M. Maffei, B. Piccirillo, C. De Lisio, G. De Filippis, V. Cataudella, E. Santamato, L. Marrucci, M. Lewenstein, and P. Massignan, Detection of Zak phases and topological invariants in a chiral quantum walk of twisted photons, Nat. Commun. 8, 15516 (2017).

[29] X.-Y. Xu, Q.-Q. Wang, S.-J. Tao, W.-W. Pan, Z. Chen, M. Jan, Y.-T. Zhan, K. Sun, J.-S. Xu, Y.-J. Han, C.-F. Li, and G.-C. Guo, Experimental classification of quenched quantum walks by dynamical Chern number, Phys. Rev. Research 1, 033039 (2019).

[30] E. Flurin, V. V. Ramasesh, S. Hacohen-Gourgy, L. S. Martin, N. Y. Yao, and I. Siddiqi, Observing Topological Invariants Using Quantum Walks in Superconducting Circuits, Phys. Rev. X 7, 031023 (2017).

[31] X.-Y. Guo, C. Yang, Y. Zeng, Y. Peng, H.-K. Li, H. Deng, Y.-R. Jin, S. Chen, D. Zheng, and H. Fan, Observation of a Dynamical Quantum Phase Transition by a Superconducting Qubit Simulation, Phys. Rev. Appl. 11, 044080 (2019).

[32] J. Li, R.-L. Chu, J. K. Jain, and S.-Q. Shen, Topological Anderson Insulator, Phys. Rev. Lett. 102, 136806 (2009).

[33] H. Jiang, L. Wang, Q.-F. Sun, and X. C. Xie, Numerical study of the topological Anderson insulator in $\mathrm{HgTe} / \mathrm{CdTe}$ quantum wells, Phys. Rev. B 80, 165316 (2009).

[34] C. W. Groth, M. Wimmer, A. R. Akhmerov, J. Tworzydło, and C. W. J. Beenakker, Theory of the Topological Anderson Insulator, Phys. Rev. Lett. 103, 196805 (2009).

[35] H.-M. Guo, G. Rosenberg, G. Refael, and M. Franz, Topological Anderson Insulator in Three Dimensions, Phys. Rev. Lett. 105, 216601 (2010).

[36] H.-C. Hsu and T.-W. Chen, Topological Anderson insulating phases in the long-range Su-Schrieffer-Heeger model, Phys. Rev. B 102, 205425 (2020).

[37] I. Mondragon-Shem, T. L. Hughes, J. Song, and E. Prodan, Topological Criticality in the Chiral-Symmetric AIII Class at Strong Disorder, Phys. Rev. Lett. 113, 046802 (2014).

[38] P. Titum, N. H. Lindner, M. C. Rechtsman, and G. Refael, Disorder-Induced Floquet Topological Insulators, Phys. Rev. Lett. 114, 056801 (2015).

[39] P. Titum, E. Berg, M. S. Rudner, G. Refael, and N. H. Lindner, Anomalous Floquet-Anderson Insulator as a Nonadiabatic Quantized Charge Pump, Phys. Rev. X 6, 021013 (2016).

[40] P. Titum, N. H. Lindner, and G. Refael, Disorder-induced transitions in resonantly driven Floquet topological insulators, Phys. Rev. B 96, 054207 (2017).

[41] H. Liu, I. C. Fulga, and J. K. Asbóth, Anomalous levitation and annihilation in Floquet topological insulators, Phys. Rev. Research 2, 022048(R) (2020).

[42] M. M. Wauters, A. Russomanno, R. Citro, G. E. Santoro, and L. Privitera, Localization, Topology, and Quantized Transport in Disordered Floquet Systems, Phys. Rev. Lett. 123, 266601 (2019).

[43] A. Rahmani and S. Vishveshwara, Interplay of Anderson localization and quench dynamics, Phys. Rev. B 97, 245116 (2018).

[44] R. Lundgren, F. Liu, P. Laurell, and G. A. Fiete, Momentumspace entanglement after a quench in one-dimensional disordered fermionic systems, Phys. Rev. B 100, 241108(R) (2019).

[45] E. J. Meier, F. A. An, A. Dauphin, M. Maffei, P. Massignan, T. L. Hughes, and B. Gadway, Observation of the topological Anderson insulator in disordered atomic wires, Science $\mathbf{3 6 2}$, 929 (2018). 
[46] Q. Niu, D. J. Thouless, and Y.-S. Wu, Quantized Hall conductance as a topological invariant, Phys. Rev. B 31, 3372 (1985).

[47] X.-L. Qi, Y.-S. Wu, and S.-C. Zhang, General theorem relating the bulk topological number to edge states in two-dimensional insulators, Phys. Rev. B 74, 045125 (2006).

[48] D. Gresch, G. Autès, O. V. Yazyev, M. Troyer, D. Vanderbilt, B. A. Bernevig, and A. A. Soluyanov, Z2pack: Numerical implementation of hybrid Wannier centers for identifying topological materials, Phys. Rev. B 95, 075146 (2017).

[49] Y. Kuno, Disorder-induced Chern insulator in the HarperHofstadter-Hatsugai model, Phys. Rev. B 100, 054108 (2019).

[50] J. Bonini, D. Vanderbilt, and K. M. Rabe, Berry flux diagonalization: Application to electric polarization, Phys. Rev. B 102, 045141 (2020).

[51] H. Li and F. D. M. Haldane, Entanglement Spectrum as a Generalization of Entanglement Entropy: Identification of Topological Order in Non-Abelian Fractional Quantum Hall Effect States, Phys. Rev. Lett. 101, 010504 (2008).

[52] F. Pollmann, A. M. Turner, E. Berg, and M. Oshikawa, Entanglement spectrum of a topological phase in one dimension, Phys. Rev. B 81, 064439 (2010).

[53] L. Fidkowski, Entanglement Spectrum of Topological Insulators and Superconductors, Phys. Rev. Lett. 104, 130502 (2010).

[54] A. M. Turner, Y. Zhang, and A. Vishwanath, Entanglement and inversion symmetry in topological insulators, Phys. Rev. B 82, 241102(R) (2010).

[55] I. Peschel and M.-C. Chung, On the relation between entanglement and subsystem Hamiltonians, Europhys. Lett. 96, 50006 (2011).

[56] T. L. Hughes, E. Prodan, and B. A. Bernevig, Inversionsymmetric topological insulators, Phys. Rev. B 83, 245132 (2011).

[57] P.-Y. Chang, C. Mudry, and S. Ryu, Symmetry-protected entangling boundary zero modes in crystalline topological insulators, J. Stat. Mech. (2014) P09014.

[58] M. McGinley and N. R. Cooper, Topology of One-Dimensional Quantum Systems Out of Equilibrium, Phys. Rev. Lett. 121, 090401 (2018).

[59] L. Pastori, S. Barbarino, and J. C. Budich, Signatures of topology in quantum quench dynamics and their interrelation, Phys. Rev. Research 2, 033259 (2020).
[60] I. Peschel, Calculation of reduced density matrices from correlation functions, J. Phys. A: Math. Gen. 36, L205 (2003).

[61] T. Kitagawa, M. S. Rudner, E. Berg, and E. Demler, Exploring topological phases with quantum walks, Phys. Rev. A 82, 033429 (2010).

[62] X. Wang, L. Xiao, X. Qiu, K. Wang, W. Yi, and P. Xue, Detecting topological invariants and revealing topological phase transitions in discrete-time photonic quantum walks, Phys. Rev. A 98, 013835 (2018).

[63] H. Obuse and N. Kawakami, Topological phases and delocalization of quantum walks in random environments, Phys. Rev. B 84, 195139 (2011).

[64] M. Zeng and E. H. Yong, Discrete-time quantum walk with phase disorder: Localization and entanglement entropy, Sci. Rep. 7, 12024 (2017).

[65] N. P. Kumar, S. Banerjee, and C. M. Chandrashekar, Enhanced non-Markovian behavior in quantum walks with Markovian disorder, Sci. Rep. 8, 8801 (2018).

[66] A. MacKinnon and B. Kramer, The scaling theory of electrons in disordered solids: Additional numerical results, Z. Phys. B 53, 1 (1983).

[67] B. Kramer and A. MacKinnon, Localization: Theory and experiment, Rep. Prog. Phys. 56, 1469 (1993).

[68] C. H. Lewenkopf and E. R. Mucciolo, The recursive Green's function method for graphene, J. Comput. Electron. 12, 203 (2013).

[69] C.-K. Chiu, H. Yao, and S. Ryu, Classification of topological insulators and superconductors in the presence of reflection symmetry, Phys. Rev. B 88, 075142 (2013).

[70] C.-K. Chiu, J. C. Y. Teo, A. P. Schnyder, and S. Ryu, Classification of topological quantum matter with symmetries, Rev. Mod. Phys. 88, 035005 (2016).

[71] T. Morimoto and A. Furusaki, Topological classification with additional symmetries from Clifford algebras, Phys. Rev. B 88, 125129 (2013).

[72] K. Shiozaki and M. Sato, Topology of crystalline insulators and superconductors, Phys. Rev. B 90, 165114 (2014).

[73] S. Kivelson, Wannier functions in one-dimensional disordered systems: Application to fractionally charged solitons, Phys. Rev. B 26, 4269 (1982). 